\title{
Modelling Axi-symmetric Travelling Waves in a Dielectric with Nonlinear Refractive Index
}

\author{
C.A. Stuart
}

\begin{abstract}
We consider an isotropic dielectric with a nonlinear refractive index. The medium may be inhomogeneous but its spatial variation has an axial symmetry. We characterize all monochromatic axi-symmetric travelling waves as solution of a system of six second order differential equations on $(0, \infty)$. Boundary conditions at 0 ensure the regularity of the fields on the axis. Guided waves satisfy additional conditions at $\infty$.Special solutions of this system correspond to what are normally referred to as TE and TM modes.
\end{abstract}

Mathematics Subject Classification (2000). 35Q60; 78A60; 34B16.

Keywords. travelling waves, nonlinear dielectric, guided waves.

\section{Introduction}

The confinement of light in a waveguide or optical fibre is usually achieved by exploiting the effect of variations in the refractive index due to inhomogeneity of the medium through which the beam is propagating. As can be understood from Snell's law and the phenomenon usually called total internal reflection, the favorable configuration consists of a region of higher refractive index surrounded by a layer, or layers, of materials having a lower refractive index, [14]. However, if the width of the region in which confinement is sought is comparable to the wavelength of the light, geometrical optics does not provide a satisfactory model and the electromagnetic theory, based on Maxwell's equations should be used, [14]. Furthermore, it is 
also well-known that nonlinear effects can be used to enhance, or even to produce, the guidance of light, see $[1,25]$. For example, no guidance occurs in a homogeneous linear medium (where the refractive index is constant) whereas it will occur, at least for sufficiently intense beams, in a homogeneous self-focusing medium (where the refractive index is an increasing function of the intensity of the light passing through it, $[7,11])$.

The mathematical discussion of this phenomenon amounts to the study of special solutions of Maxwell's equations in a medium whose constitutive relation expresses the electric displacement field as a nonlinear function of the electric field. The special solutions which have been studied in this context are either TE (transverse electric field) or TM (transverse magnetic field) modes having a planar or cylindrical symmetry. An enormous number of particular cases have been studied in the physics and engineering literature for more than twenty years now using a mixture of analytical and numerical methods, [13] for an extensive sample.

Mathematically rigorous results concerning the existence/non-existence and parameter dependence of such modes have also been established in considerable generality and we return this in the final section. However there seems to be no systematic and unified derivation of these various models from general principles which shows what the essential ingredients are and which reveals to what extent these modes constitute particular cases or exhaust all possibilities. The exposition here goes some way to acomplishing this, at least in the important context of axial symmetry that is relevant for models of optical fibres. It leads to a coupled system of six second order differential equations (see (4.13)), together with appropriate boundary conditions, whose solutions generate all axi-symmetric travelling waves that satisfy Maxwell's equations and a constitutive assumption of the type used in this area of nonlinear optics. See the end of Section 5 for a complete specification of the problem. By looking for solutions of this problem in which some of the six unknown functions are set equal to zero, one obtains the particular solutions known as TE and TM-modes. So far there seem to be no results concerning the existence and properties of more complicated solutions (hybrid modes).

In Section 2, we introduce the notions of monochromatic field, travelling wave and axi-symmetric field. Lemma 2.2 gives the general form of a field that is a monochromatic, axi-symmetric travelling wave. Since the natural representation of such fields uses cylindrical polar coordinates, some 
care has to taken when discussing the regularity of the fields on the axis of symmetry and this is dealt with in Lemma 2.3 and Corollary 2.4.

Maxwell's equations are introduced in Section 3 together with a general form for the kind of constitutive assumption that is usually adopted in the optics literature for problems concerning optical fibres and spatial solitons. This amounts to defining a nonlinear refractive index in an isotropic dielectric. Then, in Section 4, we characterize all monochromatic, axi-symmetric travelling waves that satisfy Maxwell's equations and the constitutive assumption as the solutions of a system of six second order differential equations on $(0, \infty)$ subject to some boundary conditions at 0 assuring the regularity of the fields on the axis.

Amongst all such waves, guided modes are those in which the selffocusing effect of the nonlinear refractive index balances the effects of dispersion and so the intensity of the associated beam of light remains concentrated near the axis. This notion of guidance introduces additional boundary conditions at infinity which are formulated in Section 5, leading to a complete specification of the mathematical problem. In Section 6 , we define the special classes of solutions known as TE-modes and TM-modes and we give some references to the literature concerning the rigorous analysis of these cases.

\section{Fields with special properties}

We begin with some terminology that will be used to distinguish various properties of electromagnetic fields. Let $x=\left(x_{1}, x_{2}, x_{3}\right) \in \mathbb{R}^{3}$ denote Cartesian co-ordinates in space and let $t \in \mathbb{R}$ denote time. A field $F: \mathbb{R}^{4} \rightarrow \mathbb{R}^{3}$ is monochromatic if

$$
F(x, t)=F^{1}(x) \cos \omega t+F^{2}(x) \sin \omega t \text { for } x \in \mathbb{R}^{3} \text { and } t \in \mathbb{R}
$$

for some frequency $\omega>0$ and functions $F^{1}, F^{2}: \mathbb{R}^{3} \rightarrow \mathbb{R}^{3}$. For such fields the time-average of $|F(x, t)|^{2}$ is $\frac{1}{2}\left\{\left|F^{1}(x)\right|^{2}+\left|F^{2}(x)\right|^{2}\right\}$ and this will be referred to as the intensity of the field $F$. A field $F: \mathbb{R}^{4} \rightarrow \mathbb{R}^{3}$ is a travelling wave if

$$
F(x, t)=w(x-t \xi) \text { for } x \in \mathbb{R}^{3} \text { and } t \in \mathbb{R}
$$

for some vector $\xi \in \mathbb{R}^{3} \backslash\{0\}$ and function $w: \mathbb{R}^{3} \rightarrow \mathbb{R}^{3}$. In this case $\frac{\xi}{|\xi|}$ is the direction of propagation and $v=|\xi|$ is the wave-speed. 
Lemma 2.1. Let $F: \mathbb{R}^{4} \rightarrow \mathbb{R}^{3}$ be a monochromatic field with frequency $\omega>0$ that is also a travelling wave in the direction $(0,0,1)$ with speed $v>0$. Then there exist fields $K^{1}, K^{2}: \mathbb{R}^{2} \rightarrow \mathbb{R}^{3}$ such that

$$
F(x, t)=K^{1}\left(x_{1}, x_{2}\right) \cos \left(k x_{3}-\omega t\right)+K^{2}\left(x_{1}, x_{2}\right) \sin \left(k x_{3}-\omega t\right)
$$

for all $(x, t) \in \mathbb{R}^{4}$ where $k=\frac{\omega}{v}$ is the wave-number and $\frac{2 \pi}{k}$ is the wavelength.

Proof. By assumption we have that

$$
F(x, t)=F^{1}(x) \cos \omega t+F^{2}(x) \sin \omega t \text { and } F(x, t)=w\left(x_{1}, x_{2}, x_{3}-v t\right) .
$$

Setting $x_{3}=0$ and $-v t=s$, we have that

$$
F^{1}\left(x_{1}, x_{2}, 0\right) \cos \frac{\omega s}{v}-F^{2}\left(x_{1}, x_{2}, 0\right) \sin \frac{\omega s}{v}=w\left(x_{1}, x_{2}, s\right)
$$

and so, setting

$$
K^{1}\left(x_{1}, x_{2}\right)=F^{1}\left(x_{1}, x_{2}, 0\right) \text { and } K^{2}\left(x_{1}, x_{2}\right)=-F^{2}\left(x_{1}, x_{2}, 0\right)
$$

we can write

$$
\begin{aligned}
F(x, t) & =w\left(x_{1}, x_{2}, x_{3}-v t\right) \\
& =K^{1}\left(x_{1}, x_{2}\right) \cos \frac{\omega}{v}\left(x_{3}-v t\right)+K^{2}\left(x_{1}, x_{2}\right) \sin \frac{\omega}{v}\left(x_{3}-v t\right) \\
& =K^{1}\left(x_{1}, x_{2}\right) \cos \left(k x_{3}-\omega t\right)+K^{2}\left(x_{1}, x_{2}\right) \sin \left(k x_{3}-\omega t\right) .
\end{aligned}
$$

A field $F: \mathbb{R}^{4} \rightarrow \mathbb{R}^{3}$ is axi-symmetric with respect to a line $L$ in $\mathbb{R}^{3}$ if

$$
F(\Gamma x, t)=\Gamma F(x, t) \text { for } x \in \mathbb{R}^{3} \text { and } t \in \mathbb{R}
$$

for all rotations $\Gamma$ around the axis $L$. In dealing with fields that are axisymmetric with respect to the $x_{3}$-axis, it is convenient to use cylindrical polar co-ordinates for which we adopt the usual notation

$$
\begin{gathered}
\left(x_{1}, x_{2}, x_{3}\right)=(r \cos \theta, r \sin \theta, z) \\
i_{r}=(\cos \theta, \sin \theta, 0), i_{\theta}=(-\sin \theta, \cos \theta, 0), i_{z}=(0,0,1) .
\end{gathered}
$$

Then $F: \mathbb{R}^{4} \rightarrow \mathbb{R}^{3}$ is axi-symmetric with respect to the $x_{3}$-axis if and only if

$$
\begin{aligned}
& F_{1}(r \cos \theta, r \sin \theta, z, t)=\cos \theta F_{1}(r, 0, z, t)-\sin \theta F_{2}(r, 0, z, t) \\
& F_{2}(r \cos \theta, r \sin \theta, z, t)=\sin \theta F_{1}(r, 0, z, t)+\cos \theta F_{2}(r, 0, z, t) \\
& F_{3}(r \cos \theta, r \sin \theta, z, t)=F_{3}(r, 0, z, t) .
\end{aligned}
$$

for all $r>0$ and $\theta, z, t \in \mathbb{R}$. 
Lemma 2.2. Let $F: \mathbb{R}^{4} \rightarrow \mathbb{R}^{3}$ be a monochromatic field with frequency $\omega>0$ that is also a travelling wave propagating in the direction $(0,0,1)$ with speed $v>0$. Then $F$ is axi-symmetric with respect to the $x_{3}$-axis if and only if $F$ can be expressed as

$$
\begin{aligned}
F(x, t)= & \left\{f_{r}^{1}(r) i_{r}+f_{\theta}^{1}(r) i_{\theta}+f_{z}^{1}(r) i_{z}\right\} \cos (k z-\omega t) \\
& +\left\{f_{r}^{2}(r) i_{r}+f_{\theta}^{2}(r) i_{\theta}+f_{z}^{2}(r) i_{z}\right\} \sin (k z-\omega t)
\end{aligned}
$$

for all $r>0$ and $\theta, z, t \in \mathbb{R}$ where $f_{r}^{j}, f_{\theta}^{j}, f_{z}^{j}:(0, \infty) \rightarrow \mathbb{R}$ for $j=1,2$.

Remark. A field of the form (2.9) can be written as

$$
F=F_{r} i_{r}+F_{\theta} i_{\theta}+F_{z} i_{z}
$$

where

$$
\begin{aligned}
& F_{r}=f_{r}^{1}(r) \cos (k z-\omega t)+f_{r}^{2}(r) \sin (k z-\omega t) \\
& F_{\theta}=f_{\theta}^{1}(r) \cos (k z-\omega t)+f_{\theta}^{2}(r) \sin (k z-\omega t) \\
& F_{z}=f_{z}^{1}(r) \cos (k z-\omega t)+f_{z}^{2}(r) \sin (k z-\omega t) .
\end{aligned}
$$

Proof. By Lemma 2.1 we can write $F$ in the form (2.3) and then, by (2.6) to $(2.8)$, it is axi-symmetric with respect to the $x_{3}$-axis if and only if, for $j=1,2$,

$$
\begin{aligned}
& K_{1}^{j}(r \cos \theta, r \sin \theta)=\cos \theta K_{1}^{j}(r, 0)-\sin \theta K_{2}^{j}(r, 0) \\
& K_{2}^{j}(r \cos \theta, r \sin \theta)=\sin \theta K_{1}^{j}(r, 0)+\cos \theta K_{2}^{j}(r, 0) \\
& K_{3}^{j}(r \cos \theta, r \sin \theta)=K_{3}^{j}(r, 0) .
\end{aligned}
$$

Thus

$$
K^{j}(r \cos \theta, r \sin \theta)=K_{1}^{j}(r, 0) i_{r}+K_{2}^{j}(r, 0) i_{\theta}+K_{3}^{j}(r, 0) i_{z},
$$

showing that $(2.9)$ holds with $f_{r}^{j}(r)=K_{1}^{j}(r, 0), f_{\theta}^{j}(r)=K_{2}^{j}(r, 0), f_{z}^{j}(r)=$ $K_{3}^{j}(r, 0)$.

The next result relates the smoothness of a field $F$ having the form the form (2.9) to that of its coefficients $f_{r}^{j}, f_{\theta}^{j}, f_{z}^{j}$.

Lemma 2.3. Let $f: \mathbb{R}^{3} \backslash\{(0,0, z): z \in \mathbb{R}\} \rightarrow \mathbb{R}^{3}$ be defined by (2.5) and

$$
f(x)=A(r) i_{r}+B(r) i_{\theta}+C(r) i_{z}
$$

where $A, B, C:(0, \infty) \rightarrow \mathbb{R}$. Then $f$ has a $C^{1}$-extension to $\mathbb{R}^{3}$ if and only if $A, B, C$ have $C^{1}$-extensions to $[0, \infty)$ with $A(0)=B(0)=C^{\prime}(0)=0$. 
Proof. Clearly $f \in C^{1}\left(\mathbb{R}^{3} \backslash\{(0,0, z): z \in \mathbb{R}\}\right)$ if and only if $A, B, C \in$ $C^{1}((0, \infty))$, so the only issue is the behaviour as $r \rightarrow 0$.

Let us suppose that $f$ has a $C^{1}$-extension to $\mathbb{R}^{3}$. Then $f(\cdot, 0,0) \in$ $C^{1}(\mathbb{R})$. Noting that for $s \neq 0$,

$$
f(s, 0,0)=\left(A(|s|) \frac{s}{|s|}, B(|s|) \frac{s}{|s|}, C(|s|)\right),
$$

we see that $\left.f_{1}(\cdot, 0,0)\right|_{[0, \infty)},\left.f_{2}(\cdot, 0,0)\right|_{[0, \infty)}$ and $\left.f_{3}(\cdot, 0,0)\right|_{[0, \infty)}$ are $C^{1}$-extensions of $A, B$ and $C$, respectively. Furthermore, since $f_{3}(s, 0,0)$ is an even function of $s$, we must have $C^{\prime}(0)=\left.\frac{d}{d s} f_{3}(s, 0,0)\right|_{s=0}=0$. On the other hand,

$$
\lim _{s \rightarrow 0+} f_{1}(s, 0,0)=A(0) \text { and } \lim _{s \rightarrow 0-} f_{1}(s, 0,0)=-A(0)
$$

and hence $A(0)=0$ since $f_{1}(0,0,0)=\lim _{s \rightarrow 0} f_{1}(s, 0,0)$ exists. Similarly, $B(0)=0$.

For the converse, we set $f(0,0, z)=(0,0, C(0))$ for all $z \in \mathbb{R}$ and observe that this implies that $f \in C\left(\mathbb{R}^{3}\right)$ by the properties of $A, B$ and $C$. Then, for $s \neq 0$,

$$
\lim _{s \rightarrow 0} \frac{f_{1}(s, 0, z)-f_{1}(0,0, z)}{s}=\lim _{s \rightarrow 0} \frac{A(|s|)}{|s|}=A^{\prime}(0)
$$

showing that $\partial_{1} f_{1}(0,0, z)$ exists and is equal to $A^{\prime}(0)$. Similarly,

$$
\lim _{s \rightarrow 0} \frac{f_{1}(0, s, z)-f_{1}(0,0, z)}{s}=\lim _{s \rightarrow 0} \frac{-B(|s|)}{|s|}=-B^{\prime}(0)
$$

and

$$
\lim _{s \rightarrow 0} \frac{f_{1}(0,0, z+s)-f_{1}(0,0,0)}{s}=\lim _{s \rightarrow 0} \frac{C(0)-C(0)}{s}=0
$$

so $\partial_{2} f_{1}(0,0, z)=-B^{\prime}(0)$ and $\partial_{3} f_{1}(0,0, z)=0$. Thus $\nabla f_{1}(0,0, z)=\left(A^{\prime}(0)\right.$, $\left.-B^{\prime}(0), 0\right)$ and, in the same way we see that $\nabla f_{2}(0,0, z)$ exists and is equal to $\left(B^{\prime}(0), A^{\prime}(0), 0\right)$. Furthermore,

$$
\begin{aligned}
& \lim _{s \rightarrow 0+} \frac{f_{3}(s, 0, z)-f_{3}(0,0, z)}{s}=\lim _{s \rightarrow 0+} \frac{C(s)-C(0)}{s}=C^{\prime}(0) \\
& \lim _{s \rightarrow 0-} \frac{f_{3}(s, 0, z)-f_{3}(0,0, z)}{s}=\lim _{s \rightarrow 0-} \frac{C(-s)-C(0)}{s}=-C^{\prime}(0),
\end{aligned}
$$

and, since $C^{\prime}(0)=0$, it follows that

$$
\lim _{s \rightarrow 0} \frac{f_{3}(s, 0, z)-f_{3}(0,0, z)}{s} \text { exists and is equal to } 0 .
$$


Thus $\partial_{1} f_{3}(0,0, z)=0$ and a similar discussion shows that $\partial_{2} f_{3}(0,0, z)=0$. Clearly $\partial_{3} f_{3}(0,0, z)=0$ so we have shown that $f$ is differentiable at $(0,0, z)$ with

$$
\nabla f(0,0, z)=\left(\begin{array}{ccc}
A^{\prime}(0) & -B^{\prime}(0) & 0 \\
B^{\prime}(0) & A^{\prime}(0) & 0 \\
0 & 0 & 0
\end{array}\right) .
$$

Recalling the formula

$$
\nabla f_{j}=\frac{\partial f_{j}}{\partial r} i_{r}+\frac{1}{r} \frac{\partial f_{j}}{\partial \theta} i_{\theta}+\frac{\partial f_{j}}{\partial z} i_{z}
$$

and noting that $f$ can be written as

$$
(A(r) \cos \theta-B(r) \sin \theta, A(r) \sin \theta+B(r) \cos \theta, C(r)),
$$

we find that, for $r>0$,

$$
\begin{aligned}
& \nabla f_{1}(x)^{T} \\
& =\left(\begin{array}{c}
\left\{A^{\prime}(r) \cos \theta-B^{\prime}(r) \sin \theta\right\} \cos \theta-\frac{1}{r}\{-A(r) \sin \theta-B(r) \cos \theta\} \sin \theta \\
\left\{A^{\prime}(r) \cos \theta-B^{\prime}(r) \sin \theta\right\} \sin \theta+\frac{1}{r}\{-A(r) \sin \theta-B(r) \cos \theta\} \cos \theta \\
0
\end{array}\right) \\
& =\left(\begin{array}{c}
A^{\prime}(r)+\left\{\frac{A(r)}{r}-A^{\prime}(r)\right\} \sin ^{2} \theta-\left\{B^{\prime}(r)-\frac{B(r)}{r}\right\} \sin \theta \cos \theta \\
\left\{A^{\prime}(r)-\frac{A(r)}{r}\right\} \sin \theta \cos \theta-B^{\prime}(r)+\left\{B^{\prime}(r)-\frac{B(r)}{r}\right\} \cos ^{2} \theta \\
0
\end{array}\right) \\
& \rightarrow\left(\begin{array}{c}
A^{\prime}(0) \\
-B^{\prime}(0) \\
0
\end{array}\right)=\nabla f_{1}\left(0,0, x_{3}\right)^{T} \text { as } r \rightarrow 0
\end{aligned}
$$

since $A, B \in C^{1}([0, \infty))$ with $A(0)=B(0)=0$. Similarly we find that

$$
\nabla f_{2}(x) \rightarrow\left(B^{\prime}(0), A(0), 0\right) \text { and } \nabla f_{3}(x) \rightarrow(0,0,0) \text { as } r \rightarrow \infty,
$$

showing that $\nabla f$ is continuous at $\left(0,0, x_{3}\right)$.

Using Lemma 2.3, we obtain the following criterion for the regularity of a field in the form (2.9).

Corollary 2.4. Let $F: \mathbb{R}^{4} \rightarrow \mathbb{R}^{3}$ be a field of the form (2.9). Then $F$ has a $C^{1}$-extension to $\mathbb{R}^{4}$ if and only if, $f_{r}^{j}, f_{\theta}^{j}, f_{z}^{j}:(0, \infty) \rightarrow \mathbb{R}$ have $C^{1}$ extensions to $[0, \infty)$ for $j=1,2$, with

$$
f_{r}^{j}(0)=0, f_{\theta}^{j}(0)=0, \frac{d}{d r} f_{z}^{j}(0)=0
$$




\section{Maxwell's equations in a nonlinear dielectric}

Let $E$ and $B: \mathbb{R}^{4} \rightarrow \mathbb{R}^{3}$ denote the electric and magnetic fields and let $\rho: \mathbb{R}^{4} \rightarrow \mathbb{R}$ and $J: \mathbb{R}^{4} \rightarrow \mathbb{R}^{3}$ be the electric charge and associated current densities. In cgs units, Maxwell's equations are

$$
\begin{aligned}
& \partial_{t} B=-c \nabla \wedge E \quad(1) \quad \partial_{t} E=c \nabla \wedge B-J \\
& \nabla \cdot B=0 \quad(3) \quad \nabla \cdot E=\rho
\end{aligned}
$$

where $c>0$ is speed of light in a vacuum. The energy density for the electromagnetic field is $\frac{1}{2}\left\{|E|^{2}+|B|^{2}\right\}$ and the rate at which work is done by the system is

$$
\begin{aligned}
& \frac{1}{2} \partial_{t}\left\{|E|^{2}+|B|^{2}\right\}+E \cdot J \\
& =E \cdot \partial_{t} E+B \cdot \partial_{t} B+E \cdot J \\
& =E \cdot\{c \nabla \wedge B-J\}-B \cdot c \nabla \wedge E+E \cdot J \quad \text { by }(3.1)(1) \text { and }(2) \\
& =-c \nabla \cdot(E \wedge B)
\end{aligned}
$$

where $E \wedge B$ is known as the Poynting vector. Thus, if $n: \partial V \rightarrow \mathbb{R}^{3}$ denotes the field of exterior unit normals on the boundary of a regular domain in $\mathbb{R}^{3}$, we have that

$\int_{V} \frac{1}{2} \partial_{t}\left\{|E|^{2}+|B|^{2}\right\}+E \cdot J d x=-c \int_{V} \nabla \cdot(E \wedge B) d x=-c \int_{\partial V}(E \wedge B) \cdot n d \sigma$

and so

$$
-c \int_{\partial V}(E \wedge B) \cdot n d \sigma
$$

gives the rate at which work is being done inside $V$.

A dielectric material is characterized by the following constitutive assumption:

(i) The only charges arise through the polarization of neutral atoms or molecules by the electric fields.

(ii) There is no magnetization.

(iii) The only currents arise from the movement of the charges.

From (i), there is a polarization field $P: \mathbb{R}^{4} \rightarrow \mathbb{R}^{3}$, depending on $E$, such that $\rho=-\nabla \cdot P$ and, by (ii) and (iii), $J=\partial_{t} P$. The way in which $P$ is determined by $E$ is specified by a dielectric response function. In the 
context of isotropic optical waveguides, this response is postulated to have the form

$$
P(x, t)=\chi\left(\omega, x, \frac{1}{2}\left[\left|E^{1}(x)\right|^{2}+\left|E^{2}(x)\right|^{2}\right]\right) E(x, t)
$$

for a monochromatic electric field of frequency $\omega>0$,

$$
E(x, t)=E^{1}(x) \cos \omega t+E^{2}(x) \sin \omega t
$$

where $\chi:(a, b) \times \mathbb{R}^{3} \times[0, \infty) \rightarrow \mathbb{R}$ is called the dielectric susceptibility, for some interval of frequencies $(a, b)$. (More generally, in anisotropic materials, the function $\chi$ is matrix-valued and it may depend separately on the time averages of the components of $E$. See [20, 18, 23].)

If we assume that $\chi(\omega, x, s)$ is a $C^{1}$-function of $(x, s) \in \mathbb{R}^{3} \times[0, \infty)$, then $P \in C^{1}\left(\mathbb{R}^{4}\right)$ provided that $E \in C^{1}\left(\mathbb{R}^{4}\right)$. In this case, if we define the electric displacement field $D$ by setting

$$
D=E+P,
$$

Maxwell's equations in a dielectric can be written as

$$
\begin{array}{lll}
\partial_{t} B=-c \nabla \wedge E & (1) & \partial_{t} D=c \nabla \wedge B \\
\nabla \cdot B=0 & (3) & \nabla \cdot D=0
\end{array}
$$

where

$$
D(x, t)=\left\{1+\chi\left(\omega, x, \frac{1}{2}\left[\left|E^{1}(x)\right|^{2}+\left|E^{2}(x)\right|^{2}\right]\right)\right\} E(x, t)
$$

and the function

$$
\varepsilon(\omega, x, s)=1+\chi(\omega, x, s)
$$

is known as the dielectric response.

According to the Lorentz law, the force density due to the electromagnetic fields acting on the charges is

$$
f(x, t)=\rho(x, t)\left\{E(x, t)+\frac{1}{c} v(x, t) \wedge B(x, t)\right\} .
$$

where $v: \mathbb{R}^{4} \rightarrow \mathbb{R}^{3}$ is the velocity field of the charges. By (iii), $J(x, t)=$ $\rho(x, t) v(x, t)$, and so the rate at which work is done by the fields on the charges per unit volume is

$$
f \cdot v=\rho\left\{E+\frac{1}{c} v \wedge B\right\} \cdot v=E \cdot J
$$


and furthermore

$$
\begin{aligned}
E \cdot J & =E \cdot \partial_{t} P=\chi\left(\omega, x, \frac{1}{2}\left[\left|E^{1}(x)\right|^{2}+\left|E^{2}(x)\right|^{2}\right]\right) E \cdot \partial_{t} E \\
& =\frac{1}{2} \partial_{t}\{E \cdot P\}
\end{aligned}
$$

Thus, by (3.3),

$$
\begin{aligned}
& \frac{1}{2} \partial_{t}\left\{|E|^{2}+|B|^{2}\right\}+E \cdot J \\
& =\frac{1}{2} \partial_{t}\left\{|E|^{2}+|B|^{2}\right\}+\frac{1}{2} \partial_{t}\{E \cdot P\} \\
& =\frac{1}{2} \partial_{t}\left\{E \cdot D+|B|^{2}\right\} .
\end{aligned}
$$

The rate at which work is being done inside a regular region $V$ can now be written as

$$
\int_{V} \frac{1}{2} \partial_{t}\left\{|E|^{2}+|B|^{2}\right\}+E \cdot J d x=\partial_{t} \int_{V} \frac{1}{2}\left\{E \cdot D+|B|^{2}\right\} d x
$$

and so $\frac{1}{2}\left\{E \cdot D+|B|^{2}\right\}$ is considered to be an energy density and the rate at which this energy is flowing out if $V$ is given by

$$
\begin{aligned}
& -\partial_{t} \int_{V} \frac{1}{2}\left\{E \cdot D+|B|^{2}\right\} d x=-\int_{V} \frac{1}{2} \partial_{t}\left\{|E|^{2}+|B|^{2}\right\}+E \cdot J d x \\
& =c \int_{\partial V}(E \wedge B) \cdot n d \sigma \text { by (3.2). }
\end{aligned}
$$

In particular, for monochromatic travelling waves,

$$
\begin{aligned}
& E(x, t)=E^{1}\left(x_{1}, x_{2}\right) \cos \left(k x_{3}-\omega t\right)+E^{2}\left(x_{1}, x_{2}\right) \sin \left(k x_{3}-\omega t\right) \\
& B(x, t)=B^{1}\left(x_{1}, x_{2}\right) \cos \left(k x_{3}-\omega t\right)+B^{2}\left(x_{1}, x_{2}\right) \sin \left(k x_{3}-\omega t\right),
\end{aligned}
$$

the time-average of the rate at which energy is flowing across the plane $x_{3}=d$ (constant) in the direction of propagation is

$$
\frac{c}{2} \int_{-\infty}^{\infty} \int_{-\infty}^{\infty}\left(E^{1} \wedge B^{1}+E^{2} \wedge B^{2}\right)_{3} d x_{1} d x_{2} .
$$

We observe that this quantity, known as the power, is independent of $d$ and it constitutes the fundamental measure of the strength of the beam of light associated with the travelling wave $(E, B)$. Note that, at this point, we have not yet established that there exist any monochromatic travelling waves which satisfy Maxwell's equation and (3.7). Indeed, since we have just observed that the time-average of the rate at which energy is flowing across the planes $x_{3}=d$ is independent of $d$, such waves can only exist in a 
medium that does not absorb energy. Materials of this kind are said to be transparent (or lossless) and are characterized by the additional assumption that

$$
\varepsilon(\omega, x, s) \geq A(\omega)>0
$$

about the dielectric response. The quantity $n(\omega, x, s)=\sqrt{\varepsilon(\omega, x, s)}$ is usually referred to the nonlinear refractive index. It is a function of position $x$, but depends also on the monochromatic electric field $E(x, t)$ through its frequency $\omega$ and intensity $s$. To a first approximation, the refractive index can be written as

$$
n(\omega, x, s)=n_{0}(\omega, x)+n_{2}(\omega, x) s,
$$

and this is usually referred to as a Kerr nonlinearity, [1, 25]. For a homogeneous material, there are extensive tables for the coefficients $n_{0}$ and $n_{2}$, $[11]$.

\section{Monochromatic axi-symmetric travelling waves}

As a model for an optical fibre we consider an isotropic dielectric medium. It may be inhomogeneous but the spatial variation in the dielectric response (3.7) is assumed to be axi-symmetric with respect to the $x_{3}$-axis so that, in the coordinates $(2.5)$,

$$
\varepsilon(\omega, x, s)=\mu(\omega, r, s)
$$

for some function $\mu:(a, b) \times[0, \infty) \times[0, \infty) \rightarrow \mathbb{R}$. We assume henceforth that, for $\omega \in(a, b) \subset(0, \infty)$,

(i) $\mu(\omega, \cdot, \cdot) \in C^{1}\left([0, \infty)^{2}\right)$ with

(ii) $\partial_{r} \mu(\omega, 0, s) \equiv 0$ (so that $\varepsilon(\omega, \cdot, s) \in C^{1}\left(\mathbb{R}^{3}\right)$ ) and

$$
\text { (iii) } \mu(\omega, r, s)>0 \text { for all } r, s \geq 0 \text {, }
$$

so as to ensure that the hypotheses about $\varepsilon$ introduced in Section 3 are satisfied.

We now look for solutions of Maxwell's equations that are monochromatic axi-symmetric travelling waves propagating in the direction of the $x_{3}$-axis. That is, by Corollary 2.4, we suppose that $E$ and $B$ have the form

$$
\begin{aligned}
E(x, t)= & \left\{e_{r}^{1}(r) i_{r}+e_{\theta}^{1}(r) i_{\theta}+e_{z}^{1}(r) i_{z}\right\} \cos (k z-\omega t) \\
& +\left\{e_{r}^{2}(r) i_{r}+e_{\theta}^{2}(r) i_{\theta}+e_{z}^{2}(r) i_{z}\right\} \sin (k z-\omega t)
\end{aligned}
$$


and

$$
\begin{aligned}
B(x, t)= & \left\{b_{r}^{1}(r) i_{r}+b_{\theta}^{1}(r) i_{\theta}+b_{z}^{1}(r) i_{z}\right\} \cos (k z-\omega t) \\
& +\left\{b_{r}^{2}(r) i_{r}+b_{\theta}^{2}(r) i_{\theta}+b_{z}^{2}(r) i_{z}\right\} \sin (k z-\omega t)
\end{aligned}
$$

where $e_{r}^{j}, e_{\theta}^{j}, e_{z}^{j}, b_{r}^{j}, b_{\theta}^{j}, b_{z}^{j} \in C^{1}([0, \infty))$ satisfy the geometrical boundary conditions

$$
e_{r}^{j}(0)=e_{\theta}^{j}(0)=\frac{d}{d r} e_{z}^{j}(0)=b_{r}^{j}(0)=b_{\theta}^{j}(0)=\frac{d}{d r} b_{z}^{j}(0)=0
$$

for $j=1,2$. Substituting these expression into Maxwell's equation we obtain a system of ordinary differential equations for the coefficients $e_{r}^{j}, e_{\theta}^{j}, e_{z}^{j}$, $b_{r}^{j}, b_{\theta}^{j}, b_{z}^{j}$. To write these equations more compactly we set

$$
\begin{aligned}
\mu & =\mu\left(\omega, r, \frac{1}{2}\left[\left|E^{1}(x)\right|^{2}+\left|E^{2}(x)\right|^{2}\right]\right) \\
& =\mu\left(\omega, r, \frac{1}{2} \sum_{j=1}^{2}\left(e_{r}^{j}(r)\right)^{2}+\left(e_{\theta}^{j}(r)\right)^{2}+\left(e_{z}^{j}(r)\right)^{2}\right),
\end{aligned}
$$

so that (3.6) becomes

$$
D(x, t)=\mu E(x, t) .
$$

Recall that for a $C^{1}$-field of the form (2.10) the divergence and curl can be expressed as follows.

$$
\begin{aligned}
& \nabla \cdot F=\frac{1}{r} \frac{\partial}{\partial r}\left\{r F_{r}\right\}+\frac{1}{r} \frac{\partial}{\partial \theta} F_{\theta}+\frac{\partial}{\partial z} F_{z}=\frac{1}{r} \frac{\partial}{\partial r}\left\{r F_{r}\right\}+\frac{\partial}{\partial z} F_{z} \\
& \nabla \wedge F=\frac{1}{r}\left|\begin{array}{ccc}
i_{r} & r i_{\theta} & i_{z} \\
\partial_{r} & \partial_{\theta} & \partial_{z} \\
F_{r} & r F_{\theta} & F_{z}
\end{array}\right|=-\partial_{z} F_{\theta} i_{r}+\left(\partial_{z} F_{r}-\partial_{r} F_{z}\right) i_{\theta}+\frac{1}{r} \partial_{r}\left(r F_{\theta}\right)
\end{aligned}
$$

since $F_{r}, F_{\theta}$ and $F_{z}$ are independent of $\theta$. We find that equations $\partial_{t} B=$ $-c \nabla \wedge E$ and $\partial_{t} D=c \nabla \wedge B$ reduce to the systems

$$
\left\{\begin{array} { c c c } 
{ \omega b _ { r } ^ { 1 } = } & { - c k e _ { \theta } ^ { 1 } } & { ( 1 ) } \\
{ \omega b _ { \theta } ^ { 1 } = } & { c \{ k e _ { r } ^ { 1 } + \partial _ { r } e _ { z } ^ { 2 } \} } & { ( 2 ) } \\
{ \omega b _ { z } ^ { 1 } = } & { - \frac { c } { r } \partial _ { r } ( r e _ { \theta } ^ { 2 } ) } & { ( 3 ) } \\
{ \omega b _ { r } ^ { 2 } = } & { - c k e _ { \theta } ^ { 2 } } & { ( 4 ) } \\
{ \omega b _ { \theta } ^ { 2 } = } & { c \{ k e _ { r } ^ { 2 } - \partial _ { r } e _ { z } ^ { 1 } \} } & { ( 5 ) } \\
{ \omega b _ { z } ^ { 2 } = } & { \frac { c } { r } \partial _ { r } ( r e _ { \theta } ^ { 1 } ) } & { ( 6 ) }
\end{array} \quad \text { (6) } \quad \left\{\begin{array}{ccc}
\mu \omega e_{r}^{1}= & c k b_{\theta}^{1} \\
\mu \omega e_{\theta}^{1}= & -c\left\{k b_{r}^{1}+\partial_{r} b_{z}^{2}\right\} \\
\mu \omega e_{z}^{1}= & \frac{c}{r} \partial_{r}\left(r b_{\theta}^{2}\right) \\
\mu \omega e_{r}^{2}= & c k b_{\theta}^{2} \\
\mu \omega e_{\theta}^{2}= & -c\left\{k b_{r}^{2}-\partial_{r} b_{z}^{1}\right\} \\
\mu \omega e_{z}^{2}= & -\frac{c}{r} \partial_{r}\left(r b_{\theta}^{1}\right)
\end{array}\right.\right.
$$


and the equations $\nabla \cdot B=0$ and $\nabla \cdot D=0$ to the systems

$$
\left\{\begin{array} { l l } 
{ \frac { 1 } { r } \partial _ { r } ( r b _ { r } ^ { 1 } ) + k b _ { z } ^ { 2 } = 0 } & { ( 1 ) } \\
{ \frac { 1 } { r } \partial _ { r } ( r b _ { r } ^ { 2 } ) - k b _ { z } ^ { 1 } = 0 } & { ( 2 ) }
\end{array} \quad \text { and } \left\{\begin{array}{l}
\frac{1}{r} \partial_{r}\left(r \mu e_{r}^{1}\right)+k \mu e_{z}^{2}=0 \\
\frac{1}{r} \partial_{r}\left(r \mu e_{r}^{2}\right)-k \mu e_{z}^{1}=0
\end{array}\right.\right.
$$

respectively. We observe that (4.10)(7) and (12) imply that (4.11)(3) holds and that (4.10)(9) and (10) imply that (4.11)(4) holds. Similarly any solution of the system (4.10)(1)(3)(4) and (6) satisfies (4.11)(1) and (2). Thus any $C^{1}$-solution of the system (4.10) satisfies (4.11). Of course for $C^{2}$-functions, this follows immediately from the identity $\nabla \cdot(\nabla \wedge F)=0$.

Lemma 4.1. Let $E$ and $B$ be defined by (4.5) and (4.6) where $e_{r}^{j}, e_{\theta}^{j}, e_{z}^{j}, b_{r}^{j}, b_{\theta}^{j}$, $b_{z}^{j} \in C^{1}([0, \infty))$ with

$$
e_{r}^{j}(0)=e_{\theta}^{j}(0)=\frac{d}{d r} e_{z}^{j}(0)=b_{r}^{j}(0)=b_{\theta}^{j}(0)=\frac{d}{d r} b_{z}^{j}(0)=0
$$

for $j=1,2$ and let $D$ be defined by (4.8)-(4.9). Then $E$ and $B \in C^{1}\left(\mathbb{R}^{4}\right)$. Furthermore, $E$ and $B$ satisfy (3.5) if and only if $e_{r}^{j}, e_{\theta}^{j}, e_{z}^{j}, b_{r}^{j}, b_{\theta}^{j}, b_{z}^{j} \in$ $C^{2}((0, \infty))$ and satisfy $(4.10)$.

Proof. By Corollary 2.4, $E$ and $B \in C^{1}\left(\mathbb{R}^{4}\right)$. We have already observed that $E$ and $B$ satisfy Maxwell's equations if and only if (4.10) and (4.11) are satisfied. Furthermore, if (4.10) is satisfied so is (4.11).

For any $C^{1}$-solution of the system $(4.10)$, we have that $\partial_{r}\left(e_{z}^{2}\right)=$ $\frac{\omega}{c} b_{\theta}^{1}-k e_{r}^{1}$ by $(2)$, showing that $e_{z}^{2} \in C^{2}((0, \infty))$. Similarly, the equations $(3)(5)(6)$ and $(8)(9)(11)(12)$ show that $e_{\theta}^{2}, e_{z}^{1}, e_{\theta}^{1}, b_{z}^{2}, b_{\theta}^{2}, b_{z}^{1}, b_{\theta}^{1} \in C^{2}((0, \infty))$ respectively and then (4.11) shows that $b_{r}^{1}, b_{r}^{2}, e_{r}^{1}, e_{r}^{2} \in C^{2}((0, \infty))$ since $\mu>0$ and $\mu \in C^{1}((0, \infty))$.

Remark. Under the hypotheses of Lemma 4.1, we see that if the fields $E$ and $B$ satisfy Maxwell's equations, then by substituting (4.10)(1) to (6) into (7) to (12), we see that the coefficients of the electric field satisfy the following system of six second order differential equations for $r>0$,

$$
\left\{\begin{array}{c}
\mu e_{r}^{1}=\left(\frac{c}{\omega}\right)^{2} k\left\{k e_{r}^{1}+\partial_{r}\left(e_{z}^{2}\right)\right\} \\
\mu e_{\theta}^{1}=\left(\frac{c}{\omega}\right)^{2}\left\{k^{2} e_{\theta}^{1}-\partial_{r}\left[\frac{1}{r} \partial_{r}\left(r e_{\theta}^{1}\right)\right]\right\} \\
\mu e_{z}^{1}=\left(\frac{c}{\omega}\right)^{2} \frac{1}{r} \partial_{r}\left[r\left\{k e_{r}^{2}-\partial_{r}\left(e_{z}^{1}\right)\right\}\right] \\
\mu e_{r}^{2}=\left(\frac{c}{\omega}\right)^{2} k\left\{k e_{r}^{2}-\partial_{r}\left(e_{z}^{1}\right)\right\} \\
\mu e_{\theta}^{2}=\left(\frac{c}{\omega}\right)^{2}\left\{k^{2} e_{\theta}^{2}-\partial_{r}\left[\frac{1}{r} \partial_{r}\left(r e_{\theta}^{2}\right)\right]\right\} \\
\mu e_{z}^{2}=-\left(\frac{c}{\omega}\right)^{2} \frac{1}{r} \partial_{r}\left[r\left\{k e_{r}^{1}+\partial_{r}\left(e_{z}^{2}\right)\right\}\right]
\end{array}\right.
$$


where

$$
\mu=\mu\left(\omega, r, \frac{1}{2} \sum_{j=1}^{2}\left(e_{r}^{j}(r)\right)^{2}+\left(e_{\theta}^{j}(r)\right)^{2}+\left(e_{z}^{j}(r)\right)^{2}\right) .
$$

More importantly, the converse is also true.

Proposition 4.2. Let $E$ be defined by (4.5) where $e_{r}^{j}, e_{\theta}^{j}, e_{z}^{j} \in C^{1}([0, \infty)) \cap$ $C^{2}((0, \infty))$ satisfy the system (4.13) for $r>0$ and the boundary conditions

$$
e_{r}^{j}(0)=e_{\theta}^{j}(0)=\frac{d}{d r} e_{z}^{j}(0)=0
$$

for $j=1,2$. Let $D$ be defined by (4.8)-(4.9) and let $B$ be defined by (4.6) where $b_{r}^{j}, b_{\theta}^{j}, b_{z}^{j}$ are defined by the equations $4.10(1-6)$ for $j=1,2$. Then $E$ and $B \in C^{1}\left(\mathbb{R}^{4}\right)$ and satisfy Maxwell's equations for all $(x, t) \in \mathbb{R}^{4}$.

Proof. We only have to show that the functions $b_{r}^{j}, b_{\theta}^{j}, b_{z}^{j}$ satisfy the hypotheses of Lemma 2.1. By definition $b_{r}^{j}=-\frac{c k}{\omega} e_{\theta}^{j}$ so we have immediately that $b_{r}^{j} \in C^{1}([0, \infty))$ and that $b_{r}^{j}(0)=0$. Using the equations (4.13)(1) and (4) we have that $b_{\theta}^{j}=\frac{\mu \omega}{c k} e_{r}^{j}$, showing that $b_{\theta}^{j} \in C^{1}([0, \infty))$ and that $b_{\theta}^{j}(0)=0$. By $(4.10)(3)$ and (6) we see that $b_{z}^{j} \in C([0, \infty))$ and then using $(4.10)(8)$ and (11) we see that $b_{z}^{j} \in C^{1}([0, \infty))$ with $\partial_{r} b_{z}^{j}(0)=0$.

Remark. At first sight it may seem that there should be an analogous result in which Maxwell's equations are reduced to a second order system for the coefficients $b_{r}^{j}, b_{\theta}^{j}, b_{z}^{j}$. However, this cannot be done directly since the full expression (4.8) for the response function $\mu$ contains the coefficients $e_{r}^{j}, e_{\theta}^{j}, e_{z}^{j}$. To obtain a system containing only the coefficients $b_{r}^{j}, b_{\theta}^{j}, b_{z}^{j}$, one must first invert the constitutive relation (3.6) so as to express $E$ as a function of $D$ and then use (3.5)(2) to define $D$, and then $E$, as a function of the coefficients $b_{r}^{j}, b_{\theta}^{j}, b_{z}^{j}$. Then the equation (3.5)(1) can be reduced to a second order system for the $b_{r}^{j}, b_{\theta}^{j}, b_{z}^{j}$. The invertion of the constitutive relation can be achieved under quite natural assumptions. This approach was first used in [20] and has been further exploited in $[17,19]$.

\section{Guided waves}

In the previous section we showed that all monochromatic, axi-symmetric, travelling waves in an optical fibre are obtained as solutions of a system of six second order differential equations on the interval $(0, \infty)$. However 
not all such solutions correspond to physically realistic and interesting situations. On one hand, acceptable solutions should have finite energy. Furthermore, to avoid dispersion, the intensity of the associated beam of light should be concentrated near the axis of propagation. These additional criteria are formulated as follows.

\section{Guidance conditions}

(i) The total electromagnetic energy per unit length in the direction of propagation is finite. That is,

$$
\int_{d}^{d+1} \int_{-\infty}^{\infty} \int_{-\infty}^{\infty} \frac{1}{2}\left\{E \cdot D+|B|^{2}\right\} d x_{1} d x_{2} d x_{3}<\infty \text { for all } d \in \mathbb{R} .
$$

(ii) The amplitudes of the electromagnetic fields decay to zero as the distance from the axis of propagation becomes infinite. That is,

$$
|E(x, t)| \rightarrow 0 \text { and }|B(x, t)| \rightarrow 0 \text { as } r=\sqrt{x_{1}^{2}+x_{2}^{2}} \rightarrow \infty .
$$

For fields of the form (4.5) and (4.6) the condition (i) is satisfied if and only if.

$$
\int_{0}^{\infty} r\left\{\mu \sum_{j=1}^{2}\left(e_{r}^{j}\right)^{2}+\left(e_{\theta}^{j}\right)^{2}+\left(e_{z}^{j}\right)^{2}+\sum_{j=1}^{2}\left(b_{r}^{j}\right)^{2}+\left(b_{\theta}^{j}\right)^{2}+\left(b_{z}^{j}\right)^{2}\right\} d r<\infty
$$

where, as in (4.8),

$$
\mu=\mu\left(\omega, r, \frac{1}{2} \sum_{j=1}^{2}\left(e_{r}^{j}(r)\right)^{2}+\left(e_{\theta}^{j}(r)\right)^{2}+\left(e_{z}^{j}(r)\right)^{2}\right) .
$$

Using (4.10)(1) to (6), the condition (5.1) becomes

$$
\int_{0}^{\infty} r\left\{\sum_{j=1}^{2}(1+\mu)\left[\left(e_{r}^{j}\right)^{2}+\left(e_{\theta}^{j}\right)^{2}\right]+\mu\left(e_{z}^{j}\right)^{2}+\left(\partial_{r} e_{z}^{j}\right)^{2}+\left(\partial_{r} e_{\theta}^{j}\right)^{2}+\left(\frac{e_{\theta}^{j}}{r}\right)^{2}\right\} d r<\infty .
$$

Clearly the condition (ii) amounts to

$$
e_{r}^{j}, e_{\theta}^{j}, e_{z}^{j}, b_{r}^{j}, b_{\theta}^{j}, b_{z}^{j} \rightarrow 0 \text { as } r \rightarrow \infty
$$

or using (4.10)(1) to (6),

$$
e_{r}^{j}, e_{\theta}^{j}, e_{z}^{j}, \partial_{r} e_{\theta}^{j}, \partial_{r} e_{z}^{j} \rightarrow 0 \text { as } r \rightarrow \infty .
$$


Recalling (3.8) we have that the power $P$ is

$$
\begin{aligned}
& \frac{c}{2} \int_{-\infty}^{\infty} \int_{-\infty}^{\infty}\left(E^{1} \wedge B^{1}+E^{2} \wedge B^{2}\right)_{3} d x_{1} d x_{2} . \\
& =c \pi \int_{0}^{\infty} r\left\{\sum_{j=1}^{2} e_{r}^{j} b_{\theta}^{j}-b_{r}^{j} e_{\theta}^{j}\right\} d r
\end{aligned}
$$

since

$$
E^{j}=e_{r}^{j} i_{r}+e_{\theta}^{j} i_{\theta}+e_{z}^{j} i_{z} \text { and } B^{j}=b_{r}^{j} i_{r}+b_{\theta}^{j} i_{\theta}+b_{z}^{j} i_{z}
$$

and so, using (4.10)(1) to (6), we find that

$$
P=\frac{c^{2} k \pi}{\omega} \int_{0}^{\infty} r\left\{\sum_{j=1}^{2}\left(e_{r}^{j}\right)^{2}+\left(e_{\theta}^{j}\right)^{2}\right\} d r+\frac{c^{2} \pi}{\omega} \int_{0}^{\infty} r\left\{e_{r}^{1} \partial_{r} e_{z}^{2}-e_{r}^{2} \partial_{r} e_{z}^{1}\right\} d r .
$$

Note that (5.2) implies that $P$ is finite.

We have now reached a point where we can give a complete description of the mathematical problem concerning guided axi-symmetric monochromatic travelling waves in an isotropic nonlinear optical fibre.

\section{Guided axi-symmetric travelling waves in an optical fibre}

Given a dielectric response function $\mu:(a, b) \times[0, \infty) \times[0, \infty) \rightarrow \mathbb{R}$ satisfying (4.2) to (4.4), find constants $\omega \in(a, b)$ and $k>0$ and functions $e_{r}^{j}, e_{\theta}^{j}, e_{z}^{j} \in$ $C^{1}([0, \infty)) \cap C^{2}((0, \infty))$ for $j=1,2$, such that, for $r>0$,

$$
\begin{aligned}
\mu e_{r}^{1} & =\left(\frac{c}{\omega}\right)^{2} k\left\{k e_{r}^{1}+\partial_{r} e_{z}^{2}\right\} \\
\mu e_{\theta}^{1} & =\left(\frac{c}{\omega}\right)^{2}\left\{k^{2} e_{\theta}^{1}-\partial_{r}\left[\frac{1}{r} \partial_{r}\left(r e_{\theta}^{1}\right)\right]\right\} \\
\mu e_{z}^{1} & =\left(\frac{c}{\omega}\right)^{2} \frac{1}{r} \partial_{r}\left[r\left\{k e_{r}^{2}-\partial_{r} e_{z}^{1}\right\}\right] \\
\mu e_{r}^{2} & =\left(\frac{c}{\omega}\right)^{2} k\left\{k e_{r}^{2}-\partial_{r} e_{z}^{1}\right\} \\
\mu e_{\theta}^{2} & =\left(\frac{c}{\omega}\right)^{2}\left\{k^{2} e_{\theta}^{2}-\partial_{r}\left[\frac{1}{r} \partial_{r}\left(r e_{\theta}^{2}\right)\right]\right\} \\
\mu e_{z}^{2} & =-\left(\frac{c}{\omega}\right)^{2} \frac{1}{r} \partial_{r}\left[r\left\{k e_{r}^{1}+\partial_{r} e_{z}^{2}\right\}\right]
\end{aligned}
$$

where

$$
\mu=\mu\left(\omega, r, \frac{1}{2} \sum_{j=1}^{2}\left(e_{r}^{j}(r)\right)^{2}+\left(e_{\theta}^{j}(r)\right)^{2}+\left(e_{z}^{j}(r)\right)^{2}\right)
$$


Furthermore, the solutions of this system are required to satisfy the geometrical boundary conditions

$$
e_{r}^{j}(0)=e_{\theta}^{j}(0)=\frac{d}{d r} e_{z}^{j}(0)=0,
$$

as well as the physical boundary conditions

$\int_{0}^{\infty} r\left\{\sum_{j=1}^{2}(1+\mu)\left[\left(e_{r}^{j}\right)^{2}+\left(e_{\theta}^{j}\right)^{2}\right]+\mu\left(e_{z}^{j}\right)^{2}+\left(\partial_{r} e_{z}^{j}\right)^{2}+\left(\partial_{r} e_{\theta}^{j}\right)^{2}+\left(\frac{e_{\theta}^{j}}{r}\right)^{2}\right\} d r<\infty$

and

$$
e_{r}^{j}, e_{\theta}^{j}, e_{z}^{j}, \partial_{r} e_{\theta}^{j}, \partial_{r} e_{z}^{j} \rightarrow 0 \text { as } r \rightarrow \infty .
$$

For a solution of this problem, the power, given by

$$
P=\frac{c^{2} k \pi}{\omega} \int_{0}^{\infty} r\left\{\sum_{j=1}^{2}\left(e_{r}^{j}\right)^{2}+\left(e_{\theta}^{j}\right)^{2}\right\} d r+\frac{c^{2} \pi}{\omega} \int_{0}^{\infty} r\left\{e_{r}^{1} \partial_{r} e_{z}^{2}-e_{r}^{2} \partial_{r} e_{z}^{1}\right\} d r,
$$

is the essential measure of its magnitude.

\section{TE and TM modes}

In a linear medium $\mu$ is independent of the electric field (that is, $\mu(\omega, r, s)=$ $\widetilde{\mu}(\omega, r))$ and, without loss of generality, the system (4.13) reduces to the study of four sub-problems formed by :

(I) Equation (2) alone.

(II) Equation (5) alone.

(III) The $2 \times 2$ system formed by equations (1) and (6).

(IV) The $2 \times 2$ system formed by equations (3) and (4).

For nonlinear media, all the equations in (4.13) are coupled through $\mu$, but we can still exploit its structure by looking for special solutions of the system (4.13)(1) to (6) in which some of the components of the electric field are identically zero.

\subsection{TE-modes}

We can seek solutions of (4.13) in which

$$
e_{z}^{1} \equiv e_{z}^{2} \equiv 0 \text {. }
$$

Solutions of this kind are referred to as TE-modes since the electric field is everywhere transverse to the direction of propagation. 
Using equations (4.13)(3) and (6) together with the boundary conditions this is equivalent to seeking solutions where

$$
e_{z}^{1} \equiv e_{z}^{2} \equiv e_{r}^{1} \equiv e_{r}^{2} \equiv 0 .
$$

Setting

$$
u=e_{\theta}^{1} \text { and } v=e_{\theta}^{2},
$$

this leads to the system

$$
\begin{aligned}
& u^{\prime \prime}+\frac{u^{\prime}}{r}-\frac{u}{r^{2}}-k^{2} u=-\left(\frac{\omega}{c}\right)^{2} \mu\left(\omega, r, \frac{1}{2}\left[u(r)^{2}+v(r)^{2}\right]\right) u \\
& v^{\prime \prime}+\frac{v^{\prime}}{r}-\frac{v}{r^{2}}-k^{2} v=-\left(\frac{\omega}{c}\right)^{2} \mu\left(\omega, r, \frac{1}{2}\left[u(r)^{2}+v(r)^{2}\right]\right) v
\end{aligned}
$$

for $u, v \in C^{1}([0, \infty)) \cap C^{2}((0, \infty))$ with the boundary conditions

$$
\begin{array}{r}
u(0)=v(0)=0, \\
\lim _{r \rightarrow \infty} u(r)=\lim _{r \rightarrow \infty} u^{\prime}(r)=\lim _{r \rightarrow \infty} v(r)=\lim _{r \rightarrow \infty} v^{\prime}(r)=0, \\
\int_{0}^{\infty} r\left\{(1+\mu)\left(u^{2}+v^{2}\right)+\left(u^{\prime}\right)^{2}+\left(v^{\prime}\right)^{2}+\frac{u^{2}+v^{2}}{r^{2}}\right\} d r<\infty .
\end{array}
$$

For such modes the power is

$$
P=\frac{c^{2} k \pi}{\omega} \int_{0}^{\infty} r\left\{u(r)^{2}+v(r)^{2}\right\} \quad d r<\infty .
$$

The simplest solution of this type is one in which $v \equiv 0$ (respectively $u \equiv 0$ ) and $u \in C^{1}([0, \infty)) \cap C^{2}((0, \infty))$ satisfies

$$
\begin{aligned}
u^{\prime \prime}+\frac{u^{\prime}}{r}-\frac{u}{r^{2}}-k^{2} u & =-\left(\frac{\omega}{c}\right)^{2} \mu\left(\omega, r, \frac{1}{2} u(r)^{2}\right) u \\
u(0) & =0 \\
\lim _{r \rightarrow \infty} u(r) & =\lim _{r \rightarrow \infty} u^{\prime}(r)=0 \\
\int_{0}^{\infty} r\left\{(1+\mu) u^{2}+\left(u^{\prime}\right)^{2}+\frac{u^{2}}{r^{2}}\right\} d r & <\infty
\end{aligned}
$$

and the power is

$$
P=\frac{c^{2} k \pi}{\omega} \int_{0}^{\infty} u(r)^{2} \quad r d r<\infty
$$

If $u$ is a solution of (6.9), a one parameter family of solutions of the system (6.3)(6.4) is given by setting

$$
u_{s}(r)=u(r) \cos s \text { and } v_{s}(r)=u(r) \sin s \text { for } s \in \mathbb{R}
$$


A solution of the system (6.9) to (6.12) generates a guided TE-mode whose electromagnetic fields are

$$
\begin{aligned}
& E=u(r) \cos (k z-\omega t) i_{\theta}, \\
& B=-\frac{c k}{\omega} u(r) \cos (k z-\omega t) i_{r}+\frac{c}{\omega}\left\{u^{\prime}(r)+\frac{u(r)}{r}\right\} \sin (k z-\omega t) i_{z} .
\end{aligned}
$$

The existence of axi-symmetric TE-modes is proved in $[4,6,8,15,21,24$, $26]$. The methods used to obtain some of these results are summarized in $[18]$.

\subsection{TM-modes}

Another family of special solutions is obtained by seeking solutions of (4.13) in which

$$
e_{\theta}^{1} \equiv e_{\theta}^{2} \equiv 0 .
$$

However, using the equations (4.10)(3) and (6) for the associated magnetic field, together with the boundary conditions, we see that this is equivalent to seeking solutions where

$$
b_{z}^{1} \equiv b_{z}^{2} \equiv 0
$$

and so solutions of this kind are called TM-modes since the magnetic field is now transverse to the direction of propagation. (In fact, the equations $(4.10)(1)$ and (4) show that we also have

$$
b_{r}^{1} \equiv b_{r}^{2} \equiv 0
$$

for such modes.) In this case the system (4.13) is reduced to the $4 \times 4$ system formed by the equations (1),(3),(4) and (6). The simplest solutions of this type are obtained by setting

$$
e_{r}^{1}=u, e_{z}^{1} \equiv 0, e_{r}^{2} \equiv 0 \text { and } e_{z}^{2}=v
$$

(respectively

$$
\left.e_{r}^{1}=0, e_{z}^{1} \equiv-v, e_{r}^{2} \equiv u \text { and } e_{z}^{2}=0\right)
$$


where the functions $u$ and $v$ satisfy

$$
\begin{array}{r}
k\left(v^{\prime}+k u\right)=\left(\frac{\omega}{c}\right)^{2} \mu\left(\omega, r, \frac{1}{2}\left[u(r)^{2}+v(r)^{2}\right]\right) u \\
\frac{1}{r}\left[r\left(v^{\prime}+k u\right)\right]^{\prime}=-\left(\frac{\omega}{c}\right)^{2} \mu\left(\omega, r, \frac{1}{2}\left[u(r)^{2}+v(r)^{2}\right]\right) v \\
\left.u(0)=v^{\prime} 0\right)=0 \\
\lim _{r \rightarrow \infty} u(r)=\lim _{r \rightarrow \infty} u^{\prime}(r)=\lim _{r \rightarrow \infty} v(r)=\lim _{r \rightarrow \infty} v^{\prime}(r)=0 \\
\int_{0}^{\infty} r\left\{(1+\mu) u^{2}+\mu v^{2}+\left(v^{\prime}\right)^{2}\right\} d r<\infty .
\end{array}
$$

For such solutions the power is

$$
P=\frac{c^{2} \pi}{\omega} \int_{0}^{\infty} u\left\{k u+v^{\prime}\right\} \quad r d r<\infty .
$$

As in the TE case, a one parameter family of solutions of the whole $4 \times 4$ system $(4.13)(1)(3)(4)(6)$ for TM-modes can be obtained from a solution of (6.17)(6.18). A solution of the system (6.17) to (6.21) generates a guided TE-modes whose electromagnetic fields are

$$
\begin{aligned}
& E=u(r) \cos (k z-\omega t) i_{r}+v(r) \sin (k z-\omega t) i_{z} \\
& B=\frac{c}{\omega}\left\{k u(r)+v^{\prime}(r)\right\} \cos (k z-\omega t) i_{\theta} .
\end{aligned}
$$

The existence of axi-symmetric TM-modes is proved in [10, 19, 5, 22, 23]. The methods used to obtain some of these results are summarized in [18]. For the Kerr nonlinearity (3.10), numerical simulations of system (6.17) to (6.21) are given in [3].

\section{References}

[1] R.V. Akhmanov, R.V. Khorklov and A.P. Sukhorukov, Self-focusing, selfdefocusing and self-modulation of laser beams, in Laser Handbook, edited by F.T. Arecchi and E.O. Schulz Dubois, North-Holland, Amsterdam, 1972.

[2] Y. Chen, TE and TM families of self-trapped beams, IEEE J. Quantum Elect. 27 (1991), 1236-1241.

[3] Y. Chen and A.W. Snyder, TM-type self-guided beams with circular crosssection, Electr. Lett. 27 (1991), 565-56.

[4] O. John and C.A. Stuart, Guidance properties of a cylindrical defocusing waveguide, Comm. Math. Univ. Carolinae 35 (1994), 653-673. 
[5] M. Lucia, Bifurcation problems related to special solutions of Maxwell's equations, Thèse No 1748 (1997), EPFL, Lausanne.

[6] J.B. McLeod, C.A. Stuart and W.C. Troy, An exact reduction of Maxwell's equations, Proc. Gregynogg Conference, Birkhäuser (1992), 391-405.

[7] J.F. Reintjes, Nonlinear Optical Processes, in Encyclopedia of Physical Science and Technology, Vol. 9, Academic Press, New York.

[8] H.J. Ruppen, Multiple cylindrical TE-Modes for a homogeneous self-focusing dielectric, Nonlinear World 2 (1995), 387-418.

[9] H.J. Ruppen, Multiple TE-modes for planar self-focusing wave guides, Ann. Mat. Pura Appl., IV Ser. 172 (1997), 323-377.

[10] H.J. Ruppen, Multiple cylindrical TM-modes for a homogeneous self-focusing dielectric, J. Differ. Equat. 134 (1997), 286-319.

[11] W.L. Smith, Nonlinear refractive index, Handbook of Laser Science and Technology, Vol. 3, edited by M.J. Weber, CRC Press, Boca Raton, 1986.

[12] G.I. Stegeman and M. Segev, Optical solitons and their interactions: universality and diversity, Science, 286 (1999), 1518.

[13] G.I. Stegeman, Nonlinear guided wave optics, in Contemporary Nonlinear Optics, edited by G.P. Agrawal and R.W. Boyd, Academic Press, Boston, 1992.

[14] A. W. Snyder and J. D. Love, Optical Wave Guide Theory, Chapman and Hall, London, 1983.

[15] C.A. Stuart, Self-trapping of an electromagnetic field and bifurcation from the essential spectrum, Arch. Rat. Mech. Anal. 113 (1991), 65-96.

[16] C.A. Stuart, Guidance properties of planar waveguides, Arch. Rat. Mech. Anal. 125 (1993), 145-200.

[17] C.A. Stuart, Guided TM-modes in a self-focusing anisotropic dielectric, Nonlinear Problems in Applied Mathematics, Editors T.S. Angell et al., SIAM, Philadelphia, 1995.

[18] C.A. Stuart, Cylindrical TE and TM modes in a self-focusing dielectric, Pitman Lecture Notes in Math., No 343, Longman, London, 1996.

[19] C.A. Stuart, Cylindrical TM-modes in a homogeneous self-focusing dielectric, Math. Models and Methods in Applied Scis. 6 (1996), 977-1008.

[20] C.A. Stuart, Magnetic field wave equations for TM-modes in nonlinear optical waveguides, Reaction Diffusion Systems, Editors Caristi and Mitidieri, Marcel Dekker, New York, 1997.

[21] C.A. Stuart and H.S. Zhou, A variational problem related to self-trapping of an electromagnetic field, Math. Methods in Applied Sci. 19 (1996), 1397-1407.

[22] C.A. Stuart and H.S. Zhou, Existence of guided cylindrical TM-modes in a homogeneous self-focusing dielectric, Ann. Inst. H. Poincaré, Analyse non linéaire 18 (2001), 69-96. 
[23] C.A. Stuart and H.S. Zhou, A constrained minimization problem and its application to guided cylindrical TM-modes in an anisotropic self-focusing dielectric, Calc. Var. 16 (2003), 335-373.

[24] C.A. Stuart and H.S. Zhou, Axi-symmetric TE-modes in a self-focusing dielectric, preprint 2003.

[25] O. Svelto, Self-focusing, self-trapping and self-phase modulation of laser beams, in Progress in Optics, Vol. 12, editor E. Wolf, North-Holland, Amsterdam, 1974.

[26] H.S. Zhou, A variational approach to nonlinear eigenvalue problems arising in optics, Thèse No 1749 (1997), EPFL, Lausanne.

C.A. Stuart

IACS - FSB, EPFL, CH

1015 Lausanne, Switzerland

e-mail: charles.stuart@epfl.ch

Lecture held in the Seminario Matematico e Fisico on June 13, 2003

Received: February, 2004

(10) To access this journal online:

(40) http://www.birkhauser.ch 